\title{
Applications of the PLATO computer system to research in social psychology
}

\author{
ROBERT W. HOLT \\ George Mason University, Fairfax, Virginia 22030
}

\begin{abstract}
The use of the PLATO CAI system in psychological research is discussed. This discussion covers the use of the PLATO system for research in personal space, attribution, person perception, and group decision making. The aspects of the PLATO system that facilitate research in each of these topic areas are discussed in detail. Further modifications of the PLATO system that would enhance its applicability to psychological research are also discussed.
\end{abstract}

The major advantage of the PLATO computer-aided instruction (CAI) system in executing research in psychology is the control of presented information and randomization of potential confounding variables, such as order of presentation. The major disadvantages of this system lie in the difficulty of extracting data from the study in a machine-readable form for analysis and the inevitable loss of information during a system crash. The advantages and disadvantages of the PLATO IV system in psychological research are discussed in detail by examining four different investigations completed by this author using the PLATO III and PLATO IV systems.

\section{INDIVIDUAL RESEARCH}

Three of the investigations summarized here focused on the individual. The nature of the stimuli presented and the format of presentation varied widely across these three studies, as did the measured dependent variables, since each study focused on a different theoretical area in social psychology.

\section{Personal Space Research}

The personal space study used the graphics options in PLATO and the interactive facilities of the system. There were two basic sections to this study: in one section, the person utilized graphically depicted situations and information in order to make inferences about the expected interpersonal distances between the two depicted persons; in the second section, the interpersonal distances were set for a given situation and other inferences were made about the nature of the relationship between the two depicted persons.

In this study, the PLATO graphics capability was used to construct line drawings of three different types of settings: a public setting, a semiprivate setting, and a private setting. In the first section of the study, two silhouette figures were drawn within each setting, each figure depicting either a male or a female stimulus person. In addition, the nature of the relationship between the two stimulus persons was presented in large writing at the top of the display. The important advantages of the PLATO graphics package were that (1) using the cursor-directed interactive graphics editor made construction of the stimuli quick and easy, (2) each component of the depicted displays could be accessed independently to create all possible stimulus-context combinations for presentation to the subjects, and (3) each silhouette figure could be redrawn relative to the input given by the subject on the terminal. The last advantage allowed us to program it such that the subject could press two keys on the terminal that would have the effect of moving the presented silhouette figure either toward or away from the other silhouette figure on the screen. The adjusted interpersonal distance between the two depicted figures formed the major dependent variable for the first section of the study.

The graphics in this study were facilitated by several aspects of the PLATO graphics package. First, the graphics package is fully interactive and can be accessed while editing a lesson. Second, it uses a cursor-oriented construction technique for creating these displays. Third, it writes the code for generating this display at the appropriate place in the lesson. Finally, the entire display can be moved to any place on the screen, rotated by any given angle, and sized to become larger or smaller.

PLATO contains commands that allow for random selection without replacement (RANDP) and random selection with replacement (RANDU). Here, RANDP was used to randomize the order of presentation of the entire set of possible stimulus combinations. This specific PLATO function allows random selection without replacement from a specified range of integer values for a variable. For this application, a condition variable was constructed such that each unique value of the condition variable referenced a unique combination of stimuli presented to the subject. The random selection function selected each possible value of the conditionassignment variable once and only once for each subject. Each subject, therefore, received a different random permutation of the possible orders of presentation of the stimulus conditions. In addition, this condition variable was utilized to store the data from each stimulus condition in the appropriate position of the data array 
for that subject such that the data array for each subject was hierarchically organized in the same manner. The randomization function was used similarly for all of the studies discussed in this paper.

\section{Attribution Research}

The attribution study was designed to directly and specifically investigate the selection of information prior to the attributional decision making process. We used Kelley's (1967) "cube" or analysis-of-variance model to structure the information made available to the subjects in each trial. Furthermore, we utilized Kelley's hypothesized causal patterns to construct underlying causal structures that could be either correctly or incorrectly identified by the subject. The modalities of Kelley's model that were of interest were persons, objects, and time periods. For this study, the domain of information available to the subject concerned the reactions of five hypothetical persons to five news magazines over a period of 5 weeks.

The presentation of this entire 5 by 5 by 5 set of possible information was accomplished by presenting the information in five 5 by 5 matrices on the PLATO terminal, with the rows and columns of each matrix labeled with the appropriate modality values plus a label for each matrix stating what level of the third modality it represented. Each trial contained a different "hidden" causal pattern. To prevent scanning biases from systematically confounding the selection of one modality of information vs. another, it was necessary to randomly assign within each trial the assignment of modality (persons, news magazines, weeks) to the rows, columns, and faces of the 5 by 5 by 5 matix. In addition, of course, the order of presentation of the trial conditions was also randomized without replacement.

For each trial, the subject sequentially selected elements from the available set of information elements until he felt that he had sufficient information to make an attributional decision about the "real" causes occurring in the depicted situation. Both the item of information selected and the latency of the selection (in seconds) were recorded as primary dependent variables for analysis. Since the amount of information selected for each trial varied from 0 to 125 pieces of information and formed a data record of variable length, the output from each trial was tagged with the subject's ID and the condition identifier for that trial and stored sequentially.

The advantages of PLATO for this research were (1) the ability to randomly assign modalities to rows, columns, and faces of the stimulus information cube, (2) the ability to construct the "hidden" causal pattern with different degrees of random error both in the causal pattern itself and in the "background" set of events (independently), and (3) the ability to store and identify the item selected and the time of selection for the information selected across trials.
Latency measurements on PLATO depend on the accessing of an internal clock. This accessing depends on the timesharing of the terminal and may be slightly affected by the time slice at the moment of response, depending on the amount of load on the overall system. Therefore, these latency measurements were accurate to about the nearest $.10 \mathrm{sec}$. The use of MICRO-TUTOR on a dedicated microcomputer should allow for latency estimates that would be even more precise.

\section{Person Perception}

In this study, the one aspect of PLATO utilized that has not been previously discussed was the randomaccess microfiche facility. This facility was available on the plasma-panel PLATO terminals in use at the University of Illinois but it is not presently available on the CRT-type PLATO terminals. The microfiche facility involved the back-screen projection of a microfiche image onto the plasma panel of the PLATO terminal. Some loss of resolution occurred due to the presence of the grid lines in the plasma panel and due to the extremely small size of the microfiche image. On the plus side, however, the small size of the microfiche image enabled us to put 256 pictures of stimulus persons onto one microfiche.

This study involved an attempt to isolate systematically different individual assessment schemes involved in forming an overall evaluative response to a picture of a stimulus person. PLATO was utilized to present the entire set of 256 slides of persons in a unique random order to each participant. Each participant rated various components of the stimulus person, such as his or her dress style, facial expression, body shape, and so forth. Additionally, a final overall evaluation of each slide was required. The large size of the stimulus sample was necessary in order to perform a multiple-regression analysis for each subject's data singly. Using the microfiche facility of PLATO enabled us both to present a large number of stimulus slides and to present these slides in a unique random order to each subject in an easily programmable manner. Furthermore, use of a variable pointing to the particular slide presented allowed us to store the stimulus responses in a common order for all subjects.

\section{GROUP RESEARCH}

This research involved a simulated group interaction in an extended Prisoner's Dilemma game format. PLATO was used to simulate the major interpersonal strategies of cooperation and competition for the other three members of an ostensible group. The advantage of PLATO in this application is that the responses of the simulated "others" could be made to be dependent on the subject's initial response. Furthermore, to increase "realism" of the simulated other participants, we pro- 
grammed in a message exchange capability in which each subject could send messages to the other participants. Messages were concocted for the simulated participants and sent at randomly selected trials to the real subject. Again, the subject's choice of alternative positions was recorded for each trial, as well as any "group choice" that occurred whenever the simulated group members and the real subject chose the same alternative.

The simulation of group interaction in this study was facilitated by several aspects of the PLATO system. First, PLATO has excellent string handling routines for student input. Second, the TUTOR language offers an easy simulation of written output, and this written output can be made contingent on prior student input. Finally, realism can be added by introducing variable time delays before the simulated response using the PAUSE command with a randomly set time interval.

Although we did not have real communication among the group members in this particular study, such communication would be possible using the PLATO system. Basically, the lesson would include a block of common variables that could be accessed by any of the group members using the lesson. This use of the common variables would, however, have to be carefully programmed to avoid problems with overwriting and so forth. Thus, either simulated or real interaction among terminals is possible in the PLATO system.

\section{SUMMARY}

The PLATO system has a wide variety of potential applications to different kinds of psychological research. In most cases, the research could be done in alternative ways, but the implementation of these alternative methods would be more cumbersome than utilizing PLATO. The PLATO system is quite expensive, however, and in many situations the alternative methods may be less expensive.

We feel that one of the major lacks of PLATO at the present time is a convenient and flexible method of presenting purely visual material, such as slides in a randomly accessible manner. Connection of the PLATO system to a random-access video disk would be a very desirable development from the point of view of anyone doing research that requires such visual input. Furthermore, we feel that a more convenient and flexible way to enable communication among PLATO terminals would be desirable, particularly in studies of group dynamics or other topic areas that require interpersonal interaction.

\section{REFERENCE}

Kelley, H. Attributation theory in social psychology. In D. Levine (Ed.), Nebraska Symposium on Motivation, 1967, 15 192-238. 